International Journal of Wireless \& Mobile Networks (IJWMN) Vol. 3, No. 5, October 2011

\title{
Buffer Management for Preferential DELIVERY IN OPPORTUNISTIC DELAY TOLERANT NETWORKS
}

\author{
G.Fathima ${ }^{1}$, R.S.D.Wahidabanu ${ }^{2}$ \\ ${ }^{1}$ Adhiyamaan College of Engineering, Hosur, TamilNadu, India \\ fathima_acedyahoo.com \\ ${ }^{2}$ Govt. College of Engineering, Salem, TamilNadu, India \\ drwahidabanu@gmail. com
}

\begin{abstract}
Delay Tolerant Networks (DTN) present many challenges that are not present in traditional networks. Many stem from the need to deal with disconnections which directly impacts routing and forwarding. However as these networks enable communication between wide range of devices, there are secondary problems that routing strategies may need to take care of such as to deal with limited resources like buffer, bandwidth, power. Most of the routing protocols in DTN assume that the buffer size as infinite which is not the case in reality. In resource constrained environment, buffers will run out of capacity at certain point of time. Moreover due to mobility of the nodes and limited bandwidth, it is not possible to transmit all messages a node has during the short available period of contact. Consequently an efficient buffer management policy is required under resource constrained DTNs. Further, DTNs can be used to support several asynchronous applications simultaneously. Each application may have different priority. For example, an emergency alert in monitoring application is more important than the regular data. Such environment stimulates the need to introduce priority to messages. Therefore in this paper, a policy is proposed which performs buffer management with prioritization. The proposed approach with epidemic routing is evaluated through simulation and compared with other policies. It is shown that the proposed approach results in performance improvement to epidemic routing with preferential delivery.
\end{abstract}

\section{KEYWORDS}

Delay Tolerant Networks, Opportunistic networks, Buffer management, Prioritization of messages, Delivery ratio

\section{INTRODUCTION}

Delay Tolerant Networking (DTN) is a technology which supports data transfer in challenging environments where a fully connected end to end path may never exist between a source and destination. The DTN approach is well suited for deploying applications in the developing world as it allows applications to continue operating with much less infrastructure compared to more traditional networking approaches. There are many applications that make use of DTN like (i) support to low-cost internet provision in remote or developing communities [16]. (ii) in vehicular networks (VANETs) for dissemination of location dependent information (eg., local ads, traffic reports, parking information [17]). (iii) in noise monitoring, earth quake monitoring. The details of Delay Tolerant Network architecture are available in [11]. From the literature survey [9], [13], [15], [22], [24], [25] it is understood that a large amount of research has been performed in developing efficient routing algorithms for DTNs. However, it is observed that flood-based routing protocols perform poorly when resources like buffer and bandwidth are limited.

DOI : 10.5121/ijwmn.2011.3502 
International Journal of Wireless \& Mobile Networks (IJWMN) Vol. 3, No. 5, October 2011

DTNs operate with the principle of store, carry and forward. In order to cope with long disconnection, messages must be buffered for long period of time. It implies that intermediate nodes require enough buffer space to store all messages that are waiting for future communication opportunities. Moreover to achieve high delivery probability, messages are replicated to each and every node they encounter. The combination of long term storage and extensive message replication performed often by many DTN routing protocols imposes a high storage overhead on wireless nodes. Moreover, bundles which are application-level data units can often be large. In this context, it is evident that buffers will run out of capacity at certain point of time.

The next important resource is the contact capacity. i.e, how much data can be exchanged between nodes. This depends on both link technology and the duration of contact. Even if duration is precisely known, it may not be possible to predict the capacity due to fluctuation in the data rate. When the number of messages to be transmitted is very small compared to the capacity of the contact in the networks then, all messages will get transmitted when nodes come in contact with each other. Here the order of transmission is not an issue. But if the number of messages to be transmitted is more than the capacity of contact then, it is not possible to transmit all messages.

Further, transmission takes place when nodes come into each other's communication range. The node has to decide which of the messages to be transmitted among the messages those are available in the buffer. Despite inherent delay tolerance of most DTN driving applications, there can be situations where some messages may be more important than the other. For e.g., in VANETs it is reasonable to assume that an accident notification message is more important than a chat message or advertisements of nearby shops. Under such requirement, a different forwarding policy will be needed to serve different types of traffic. Consequently it would be necessary to prioritize messages and ensure that they get best possible service. So given the network limitations, the key question to be answered is, how to prioritize the messages and schedule them so that messages are delivered preferentially. In this paper, an adaptive buffer management policy with prioritization is proposed which takes care of both: which messages are to be transmitted when a new contact arises and which messages are to be dropped when buffer is full. The proposed policy does selective dropping and scheduling. It considers the lifetime as well as the priority of the messages in making such decisions. The proposed approach with epidemic routing is evaluated through simulation and compared with other policies. It is shown that the proposed approach results in performance improvement to epidemic routing with preferential delivery.

This new policy is evaluated using ONE simulator and compared with other dropping and forwarding policies. ONE is an opportunistic Network Environment Simulator which is designed specifically for DTN environment. The remainder of this paper is organized as follows: Section 2 gives a background of DTN routing mechanism and the previous work related to buffer management is discussed. Section 3 discusses about the proposed method of buffer management. The simulation setup and the results are discussed in section 4 . Section 5 concludes the paper.

\section{BACKGROUND AND RELATED WORK}

Comprehensive study of different routing mechanism is important to understand the design of DTNs. There are various routing protocols available for DTN, the details of which are available in [9], [13], [15]. They differ in the knowledge that they use in making routing decisions and the number of replication they make. The various DTN protocols are Direct Delivery, First Contact, Epidemic [4], [5], Spray and Wait [3], [21], PRoPHET [2], and MaxProp [10] routing. Among the above mentioned protocols, the first four protocols are simple routing protocols which do 
not require any knowledge about the network. The latter two protocols use some extra information to make decisions on forwarding. Further based on the replication, the DTN routing protocols can be classified as those that replicate multiple copies and those that forward only a single copy. The protocols like Direct Delivery and First Contact routing are single copy protocols where only one copy per message is routed. Therefore, the buffer requirement and their utilization is less in these protocols. The protocols like Epidemic, Spray \& Wait and PRoPHET routing are multi-copy protocols and therefore they require more buffer space and their utilization is observed to be maximum. This is an interesting case where much research is to be carried out to yield good performance when the resources are constrained. The comparison of various DTN routing protocols and their buffer utilization has been discussed in [26].

It is necessary to understand the impact of buffer size on performance, as this resource is limited in reality. Epidemic routing is chosen as baseline for evaluation as this routing is based on flooding and requires huge buffer space. Epidemic routing floods each message throughout the network through its neighbours to achieve high delivery probability. As it relies on buffer to have a copy of every message at every node, buffer size has significant impact on delivery probability. A number of studies also have clearly shown that Epidemic routing has minimum delivery delay under no buffer and bandwidth constraints but performs poorly under constrained environments. The studies in [5] illustrate how the buffer constraints affect the performance of DTN routing severely.

Buffer management is a fundamental technology which controls the assignment of buffer resources among different traffic classes and aggregation of the same according to certain policies. An efficient buffer management policy is required to decide at each step which of the messages are to be dropped when buffer is full and which of the messages are to be transmitted when bandwidth is limited irrespective of the routing algorithms used. Table 1 shows the existing replication based DTN routing protocols and their assumption on availability of buffer and bandwidth; both being either limited or unlimited.

Table 1. Assumption of DTN Routing Protocols

\begin{tabular}{|l|l|l|}
\hline DTN Routing Protocols & Buffer & Bandwidth \\
\hline Epidemic, Spray \& Wait & Unlimited & Unlimited \\
\hline ProPHET & Limited & Unlimited \\
\hline MaxProp & Limited & Limited \\
\hline RAPID & Limited & Limited \\
\hline $\begin{array}{l}\text { Optimal Buffer Management (with } \\
\text { Epidemic Routing) }\end{array}$ & Limited & Limited \\
\hline Prioritized Epidemic Routing & Limited & Limited \\
\hline
\end{tabular}

The protocols like Direct Delivery and First Contact routing are single copy protocols where only one copy per message is routed in FCFS order. i.e., the messages are transmitted in the order in which they were stored in the buffer. Among the multi-copy protocols, epidemic and Spray \& Wait routing also uses FCFS forwarding policy. PRoPHET routing makes forwarding decision based on delivery predictability of the destination. It needs history of past encounters for calculation of delivery predictability. MaxProp routing assigns priorities to the messages based on hop count and delivery likelihood. Estimation of delivery likelihood is done based on historical data. It forwards the messages with high priorities when a contact arises. RAPID protocol [7] derives the per-packet utility function from administrator-specified routing metric. It forwards the messages with highest utility value first. Similarly, the Optimal policy in [18], 
International Journal of Wireless \& Mobile Networks (IJWMN) Vol. 3, No. 5, October 2011

[20], [23] derives per-message utility function from statistical learning and the message with smallest utility is dropped when the buffer is full and message with highest utility is scheduled first for transmission. In Prioritized Epidemic Routing [19], each bundle is assigned a drop priority and transmit priority which is based on hop count. i.e., the number of hops the bundle has traversed thus far. The transmission and dropping is done based on the priority. The approach presented in this paper differs from the above mentioned works in considering the traffic class and lifetime of the bundles.

The simple dropping policy used in many networks is Drop tail policy. Apart from drop tail policy there are other policies proposed in the literature [1], [6] where an arriving packet is always accepted if there is an empty buffer. Else it is accepted by dropping another packet. In general, policies which can accept an arriving packet by dropping another packet from the system are known as push - out policy. Such policies are Drop First, Drop Last, and Drop Random. Upon arrival of a packet the system can decide to either accept the packet or reject it or accept it and drop another packet based on the policy. Therefore the goal is to determine the policy which maximizes the overall throughput or equivalently minimize the overall loss probability of high priority messages.

Though a number of scheduling policies are possible, FCFS is the simple policy which is easy to implement. As long as the contact duration is long enough to transmit all messages a node has, FCFS is a very reasonable policy. However if the contact duration is limited, the policies, FCFS and drop tail are sub-optimal as it does not provide any mechanism for preferential delivering or storing of high priority messages. Considering the above said problems, the proposed policy attempts to differentiate traffic based on Class-of -Service $(\mathrm{CoS})$ and provide better levels of service in a best-effort environment. Thus the proposed policy is more advantageous in emergency applications as it does preferential delivery.

\section{Proposed System}

\subsection{Motivation}

Most of the existing routing protocols offer best effort service. There is one fundamental limitation of best effort method being used: it makes no attempt to differentiate between the traffic classes that are generated by different hosts. But to provide different services to different applications, it is necessary to differentiate traffic classes. . The unpredictable and bursty nature of DTN makes it necessary to manage the buffer. A final motivation for adding a service class to the DTN is to provide a means by which applications that are not intrinsically delay tolerant can still be supported by DTN deployment. More specifically, some applications that use DTN service require preferential delivery of certain messages. For e.g, field agents wish to communicate their findings, regarding environment hazards to other field agents which are more important than the regular findings. Moreover there may be some messages in the buffer whose lifetime is small and retaining them may not be useful as the time of next available contact is not known. Therefore a buffer management mechanism is required which is capable of differentiating the traffic and to transmit and drop messages so as to maximize the delivery ratio or minimize end to end latency.

\subsection{Network Model}

The network that is considered can be characterised as partially connected with low node density and high node mobility. The movement inherent in the nodes themselves is exploited to deliver the messages when the network is partially connected which is referred as opportunistic DTN. It is assumed that no knowledge about the network is known a priori and no infrastructure exists to provide connectivity. Assume that $\mathrm{N}$ is the total number of nodes in the network. Each of these nodes has a buffer, which can store either messages belonging to other nodes or 
messages generated by itself. Each message is destined to one of the nodes in the network and has a Time-To-Live (TTL) value. Once the TTL value expires, the message is no more useful to the application and it is dropped from the buffer. In the context of DTN, message transmission occurs only when nodes encounter each other. Consider a node which acts as an intermittent to several flows. That is the messages from several senders enter the node at various instances. Since node mobility is assumed, the node may accept messages either from other routing nodes or directly from senders. In the first case, the messages arrive back to back with constant inter arrival times and in the second case in a stochastic manner. The node then has to keep them until a connection opportunity occurs or until its storage space is full.

\subsection{Queue Model}

In DTN, bundle protocol is used for transfer of messages. A bundle is a protocol data unit of the DTN bundle protocol [8]. Bundle Processing Control Flags Bit is used to differentiate the traffic through Class-of-Service (CoS) field. The Lifetime field available in the primary bundle block gives the expiration time. This information is used for prioritization.

It is assumed that there are three priority classes of traffic: bulk, normal and expedited. Bulk messages have lowest priority, normal messages have medium priority and expedited messages have high priority. The policy gives preference to expedited messages. In the proposed approach, the available buffer is divided into many queues to hold the incoming bundles. Separate queue is maintained for each class of service as shown in Figure 1. At this point the size of the queues is not determined. Their sizes can be either defined at the beginning or varied as the correlation of traffic changes. The goal is to determine how the buffers are best shared among messages of different classes, so that the overall delivery ratio is maximized.

Assume that each node has a buffer $B$ of size $b=n(B)$ which is logically divided into three queues: $\mathrm{B}_{1}, \mathrm{~B}_{2}, \mathrm{~B}_{3}$ to accommodate high, medium and low priority bundles respectively such that $B=\left\{B_{1} \cup B_{2} \cup B_{3}\right\}$. The size of $B_{1}, B_{2}, B_{3}$ is $b_{1}, b_{2}, b_{3}$ respectively such that $b=b_{1}+b_{2}+b_{3}$. To avoid the complete negligence of medium and low priority traffic, a minimum size $\mathrm{q}_{\min }$ is reserved for medium and low priority queues. The value of $\mathrm{q}_{\min }$ is set dynamically according to the requirements of the application.

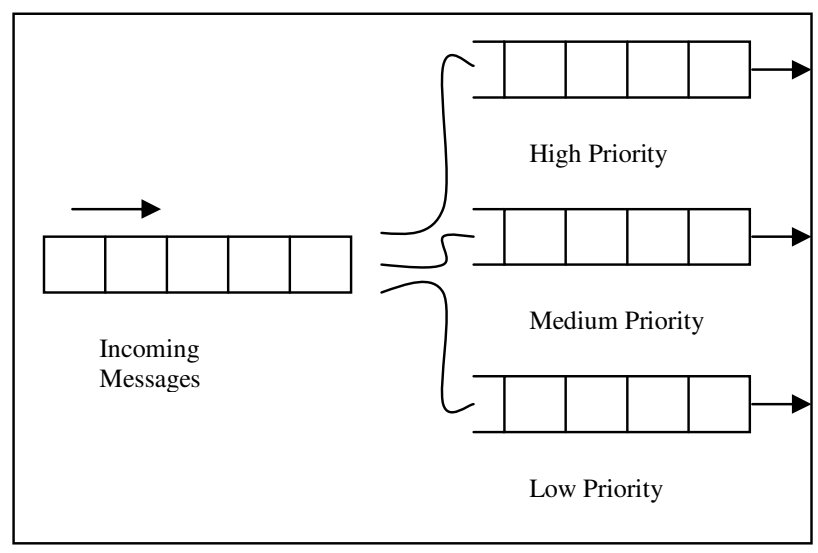

Figure 1. Maintaining Priority Queue 


\subsection{Buffer Management System}

The proposed system comprises of (i) Bundle classifier which classifies the bundles according to their priority as soon as they arrive and stores them in appropriate queue, (ii) Bundle scheduler which is invoked when the contact opportunity arises and schedules the bundles based on the policy (iii) Bundle dropper which is invoked when buffer is full and drops the bundle according to the policy. This system is illustrated in Figure 2.

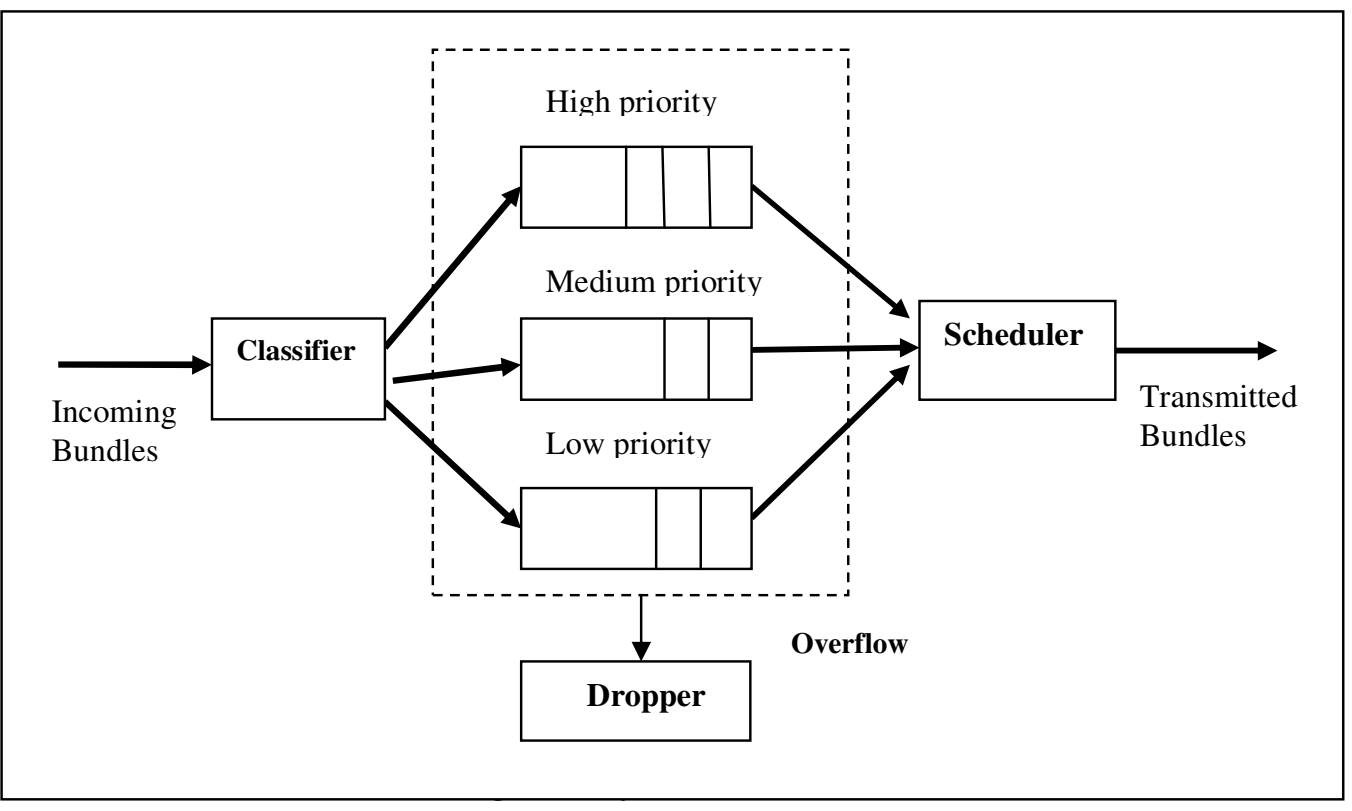

Figure 2. System Overview

\section{Bundle classifier}

Each bundle in the buffer has a set of information stored with it such as source id, traffic class and Time-To-Live(TTL). Initially bundles are classified based on their Class of Service and stored in appropriate queue as and when they arrive, by the Bundle Classifier. The service class can be specified by the application. The Bundle Classifier is a function of newly arrived bundle $b_{\text {new }}$ such as

$$
\mathrm{f}\left(\mathrm{b}_{\text {new }}\right)= \begin{cases}B_{1} \cup\left\{b_{\text {new }}\right\} & \text { if } b_{\text {new }} \text { is of high priority } \\ B_{2} \cup\left\{b_{\text {new }}\right\} & \text { if } b_{\text {new }} \text { is of medium priority } \\ B_{3} \cup\left\{b_{\text {new }}\right\} & \text { if } b_{\text {new }} \text { is of low priority }\end{cases}
$$

where $B_{1}$ contains $\left\{b_{11}, b_{12}, \ldots, b_{1 b 1}\right\}$ which are high priority messages, $B_{2}$ contains $\left\{b_{21}, b_{22}, \ldots\right.$, $\left.b_{2 b 2}\right\}$ which are medium priority messages, $B_{3}$ contains $\left\{b_{31}, b_{32}, \ldots, b_{3 b 3}\right\}$ which are low priority messages.

\section{Bundle dropper}

When the entire buffer is full, some of the bundles should be dropped to give room for new bundles. So once the buffer is full, the Bundle dropper is invoked. The Bundle dropper drops the low and medium priority bundles to give room for high priority bundles. A bundle is dropped 
automatically when the TTL expires. It is also taken care that a node should not drop its own bundle (source) to give room for newly arrived bundles. The idea of giving priority to source bundles has been proposed in [19], and was shown to improve the average delivery ratio. So the same idea is followed here. Bundle dropping is a function which identifies the bundle to be dropped according to the proposed policy. The bundle drop procedure is as follows:

Case 1:

When high priority bundle arrives, the bundle to be dropped $b_{\text {drop }}$ is identified as follows:

$\left\{\begin{array}{l}\text { if } b_{3}>q_{\min }, b_{\text {drop }} \in B_{3} \text { with least TTL value } \\ \text { else if } b_{2}>q_{\min }, b_{\text {drop }} \in B_{2} \text { with least TTL value } \\ \text { else } b_{\text {drop }} \in B_{1} \text { with least TTL value }\end{array}\right.$

Case 2:

When medium priority bundle arrives, the bundle to be dropped $b_{\text {drop }}$ is identified as follows:

$\left\{\begin{array}{l}\text { if } b_{3}>q_{\min }, b_{\text {drop }} \in B_{3} \text { with least } T T L \text { value } \\ \text { else } b_{\text {drop }} \in B_{2} \text { with least TTL value }\end{array}\right.$

Case 3:

When Low priority bundle arrives, the bundle to be dropped $b_{\text {drop }}$ is identified as follows:

$b_{\text {drop }} \in B_{3}$ with least TTL value

\section{Bundle scheduler}

When two nodes come into the communication range of each other, they start exchanging messages. Short duration of contact between the nodes and finite bandwidth may not allow the node to transmit all the messages that are available in the buffer. In such cases the order in which the messages are transmitted is significant. Bundles are transmitted according to their priority and ordered based on expiration time. Bundle scheduler transmits the bundles from high priority to low priority in a round robin fashion. The bundle to be transmitted $b_{\text {sch }}$ is identified as follows:

until nodes are in communication range

while high priority queue is not empty, $b_{\text {sch }} \in B_{1}$

$\left\{\right.$ while medium priority queue is not empty, $b_{\text {sch }} \in B_{2}$

while low priority queue is not empty, $b_{\text {sch }} \in B_{3}$

It should be noted that irrespective of the scheduling policy adopted, the messages whose destination encountered are the first to be transmitted and the same may be deleted from the buffer. Nodes do not delete messages that are forwarded to other nodes (i.e., not to destination) as long as there is sufficient space available in the buffer. 
Performance of DTN is measured in terms of average delivery ratio and average delivery delay. The average delivery ratio is defined as ratio of number of messages delivered to the destination and the total number of messages sent by the sender. The average delivery delay is measured as the average of the time taken to reach from source to destination by all messages. Both the metric equations are shown below:

Delivery ratio $=$

Number of messages delivered

Total number of messages sent by the sender

Delivery delay $=$ Average $($ the time taken to reach from source to destination by all messages)

\section{SIMULATION RESULTS AND ANALYSIS}

\subsection{Simulation Environment}

To evaluate the proposed scheme, the ONE Simulator [14] has been used. ONE is an Opportunistic Network Environment simulator which is designed specifically for DTN environment. It is a discrete event based simulator. It is a Java-based tool which provides DTN protocol simulation capabilities in a single framework. A detailed description of this simulator is available in [12]. The Mobility model used is Random Way Point (RWP) model. It is the model in which nodes move independently to a randomly chosen destination. As the network with random behaviour is considered, Epidemic routing is used as the routing algorithm.

The simulation environment consists of sparsely distributed mobile nodes and they communicate when they are in the communication range of one another. The settings of the group of nodes like buffer size transmit range, transmit speed, group speed, wait time, number of nodes in the group are set as mentioned in the Table 2.

Table 2. Parameters

\begin{tabular}{|l|l|}
\hline Parameters & Values \\
\hline Number of Nodes & 100 \\
\hline Transmit Range(m) & 250 \\
\hline Transmit speed (Mbps) & 2 \\
\hline Node Speed (km/hr) & $10-60$ \\
\hline Message size (MB) & $1-2$ \\
\hline TTL of message (min) & 30 \\
\hline Buffer size (MB) & 15 \\
\hline Simulation Time (s) & 43000 \\
\hline
\end{tabular}

\subsection{System Evaluation and Analysis}

The performance of epidemic routing under different buffer management policies is compared in terms of metrics like delivery probability and average delivery latency. Simulation results for different dropping policies with respect to delivery probability and delivery delay are shown in the Figure 3 and Figure 4 respectively. The different dropping policies that are compared are Drop Old (DO), Drop Young (DY), Drop Random (DR) and the Prioritized Policy (PP). It can 
International Journal of Wireless \& Mobile Networks (IJWMN) Vol. 3, No. 5, October 2011

be observed from the result in Figure 3 that as and when the traffic load increases, the delivery probability decreases. At the same time it also shows that there is not much difference in delivery ratio upon incorporating the new prioritized policy compared with other policies. But the prioritized policy guarantees the delivery of high priority messages first. Similarly it can be inferred from the result in Figure 4 that the delivery latency increases rapidly irrespective of the policies. But the proposed policy guarantees the delivery of high priority messages with least delay.

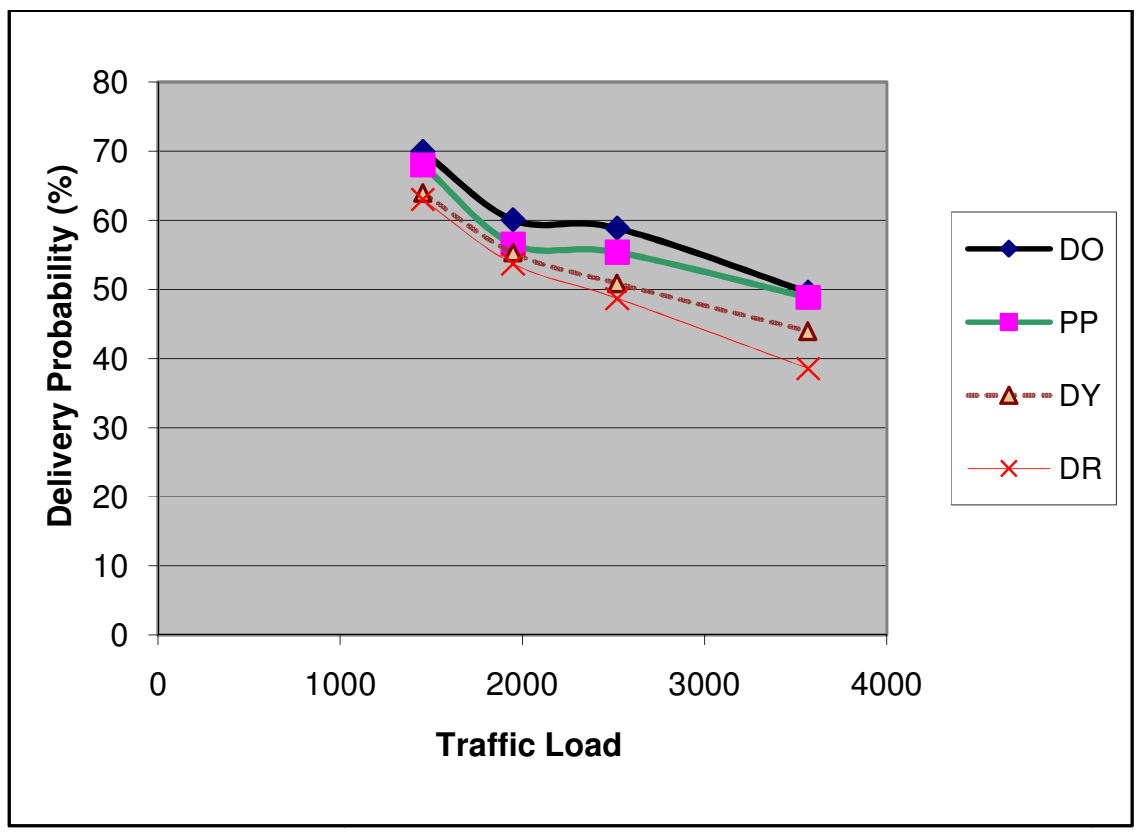

Figure 3. Delivery Probability as a function of Load

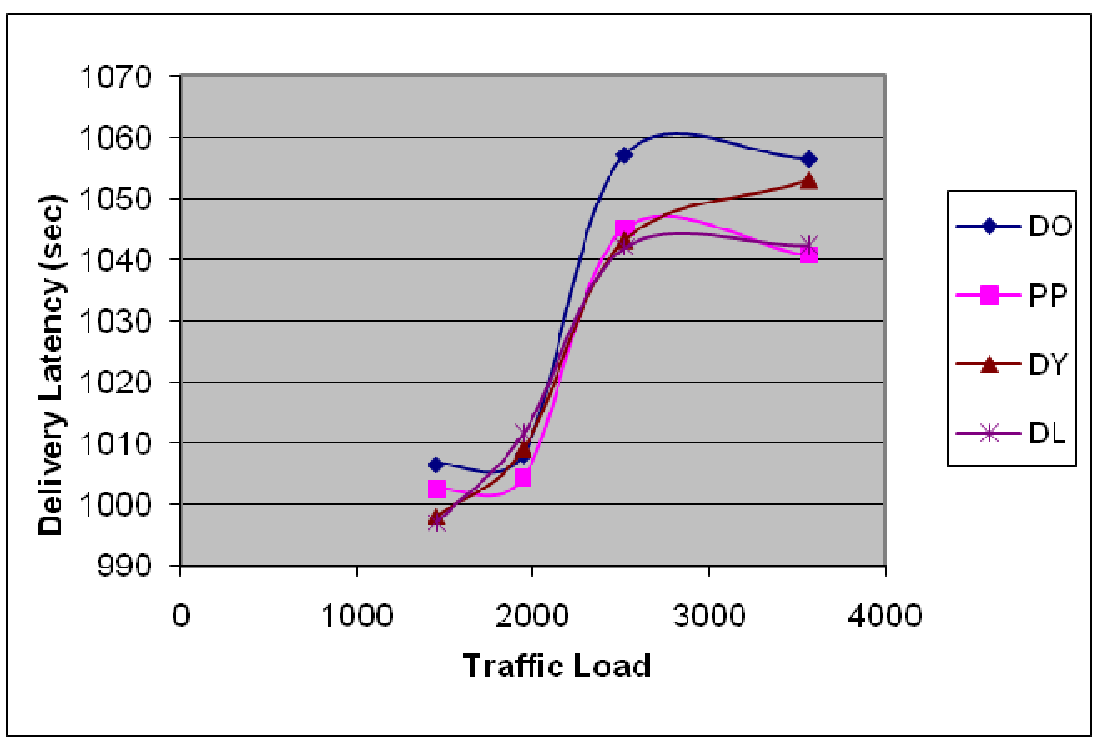

Figure 4. Delivery delay as a function of Load 
It has been shown in [1] that DO policy gives better performance in terms of delivery ratio among the different drop policies in epidemic routing. The simulation results shown in Figure 3 support it. The rationale behind this result is that an old message is likely to be duplicated to more nodes and discarding a copy of it may not decrease the overall delivery ratio much. The new prioritized policy which combines the lifetime and the priority of the messages do not decrease the delivery ratio compared to DO policy but guarantees delivery of high priority messages first. The rationale behind this result is that messages with less remaining lifetime may get automatically removed from the buffer when the lifetime expires. So forcing such messages to drop will not decrease the delivery ratio. Moreover messages with high priority are forwarded first. So they have more chances of earliest delivery than other messages. The system is further evaluated to check the performance behaviour of different priority messages at different rate of generation which is discussed in the following section.

\section{Scenario 1:}

In scenario 1, messages with different priorities are generated at equal rate and their delivery probability is observed. Here, the buffer size is set to unlimited to accommodate all messages and bandwidth is limited. The result shown is the average of several simulation runs. The result in the graph of Figure 5 confirms that the delivery probability of high priority messages is higher than other messages. This is because according to the proposed policy, all high priority messages are scheduled first. Therefore almost all messages with high priority reach their destination before the TTL expires. The result when buffer size is limited is not shown here due to space limitations. In the result, the delivery probability of low priority messages is affected due to short duration of contact and bandwidth restrictions.

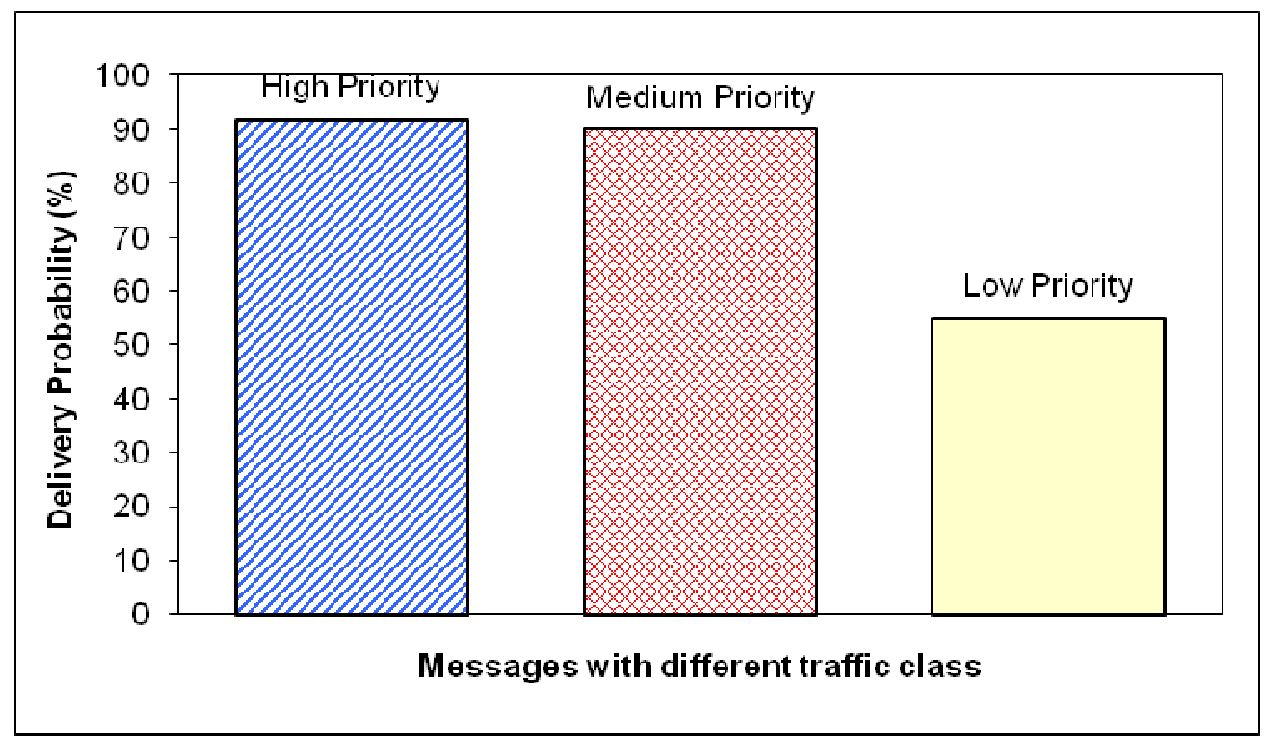

Figure 5. Delivery Probability at scenario-1

\section{Scenario 2:}

In scenario 2, messages are generated such that rate of high priority messages is more than the rate of low \& medium priority messages (i.e., high priority messages are doubled that of low and medium priority messages). In scenario 2 and 3, the bandwidth and the buffer size are set to 
be limited. When the load of high priority messages is increased, the delivery probability of low priority messages gets decreased. It can be observed in the result of Figure 6 . The rationale behind this result is that only low and medium priority messages are dropped to give room for high priority messages when there is an overflow. Moreover due to bandwidth limitations, almost all high priority messages are transmitted and only few low priority messages are transmitted. Therefore their delivery probability decreases.

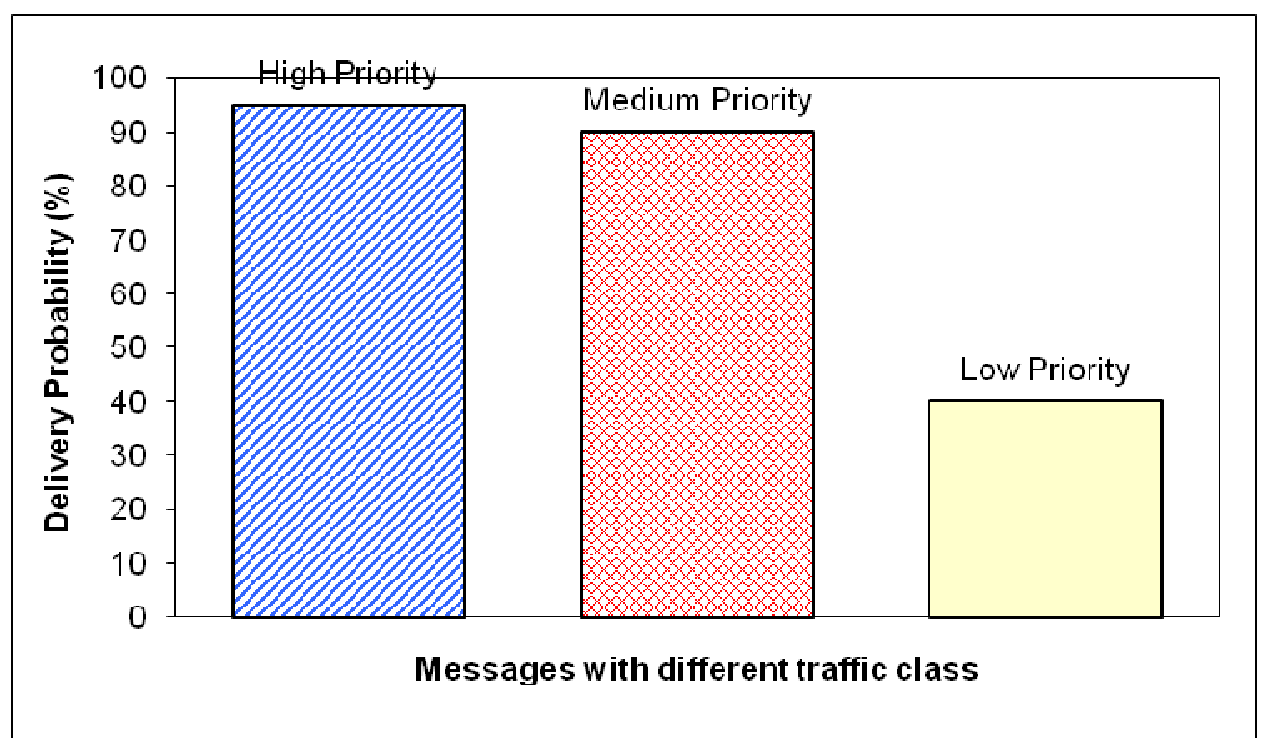

Figure 6. Delivery Probability at scenario-2

\section{Scenario 3 :}

In scenario 3, messages are generated such that rate of high priority messages is less than the rate of low \& medium priority messages (i.e, high priority messages are halved that of low \& medium priority messages). Even when there is increase in traffic load of medium and low priority messages, the delivery probability of high priority messages is not affected and remains higher. The rationale behind this result is that, as high priority messages are less, the overflow is due to only low and medium priority messages. In this scenario according to the policy, only low priority messages are dropped to give room for medium priority messages. Therefore the delivery probability of high and medium priority messages is not affected and the delivery probability of low priority messages is much lesser. It is inferred from the result shown in Figure 7.

It is observed that if a bit of low priority message loss is compromised, then high priority message loss can be reduced up to a greater extent. This improvement of the performance of high priority message loss at a little compromise of low priority message loss gives a special merit to the proposed policy. This ensures that the high prioritized traffic is forwarded with least delay and least likelihood of being dropped due to buffer overflow. It has the limitation that when the proportion of high priority messages is increased rapidly, high priority messages are less likely to get a low priority message to push out and that causes high priority loss to increase. Practically number of high priority messages will be a limited one. However using proposed policy one can alliance order of magnitude improvement in the high priority performance at the cost of moderate medium and low priority performance. 


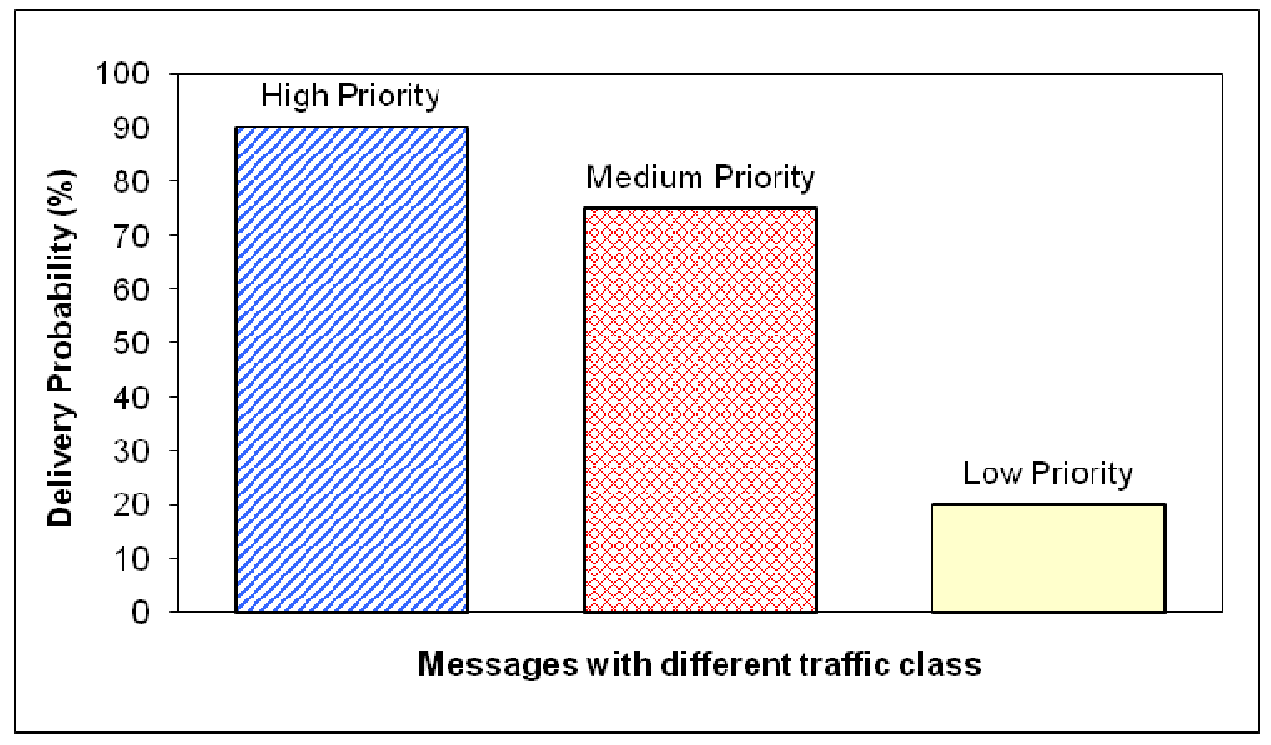

Figure 7. Delivery Probability at scenario-3.

\subsection{Merits of the System}

The work targets on application that requires preferential delivery in opportunistic DTN environment. By providing differentiated service based on the class, the best effort service has been enhanced. When compared to other approaches of [18], [19], [20], [23], the proposed approach has the credit of state less approach that minimizes the need for nodes in the network to remember anything about flows. It makes the proposed approach more practical to implement and more scalable. The messages are marked in a way that describes the service level that they should receive. Moreover it has less overhead than other approaches as there is no exchange of control traffic before bundle exchange. Since the approach is successful for opportunistic environment which is non-deterministic in nature, it can be made easily applicable for deterministic DTN. The proposed approach is more advantageous when there is strict constrains on resources like buffer and bandwidth.

\section{CONCLuSion}

Most of the DTN routing protocols operate with the assumption of infinite buffer and bandwidth. However, these resources are limited in a realistic environment. Moreover in this paper, the DTN environment considered is highly mobile and opportunistic in nature which limits the duration of contact. Therefore the work focused on effective buffer management. The approach presented in this paper prioritizes the traffic based on Class-of-Service and expiration time and performs the scheduling and dropping based on priority. It satisfies the applicationspecific requirements as the service required can be specified by the application. The proposed approach is validated through simulation. The results illustrate that the approach presented performs more or less equally to other policies in terms of delivery ratio with preferential delivery of high priority messages. The proposed policy is more suitable and advantageous in strict resource constrained environment with emergency applications. So it can be used in vehicular networks where accident notification is more important than other messages. The Fair queuing with dynamically assigned weights, can be utilized for controlling the quality of service. Thereby it addresses the integration of QoS in the DTN framework providing a bound on performance metrics like delay or throughput. Apart from delivery ratio, the other metrics like loss probability and power consumption can be considered for optimization. Finally, the 
International Journal of Wireless \& Mobile Networks (IJWMN) Vol. 3, No. 5, October 2011

work can be extended by considering energy consumed during the transmission of the messages which is carried as future work.

\section{REFERENCES}

[1] A. Davids, A. H. Fagg, and B. N. Levine, (2001) "Wearable Computers as Packet Transport Mechanisms in Highly- Partitioned Ad- Hoc Networks,” Proc. Int'l. Symp Wearable Comp., Zurich.pp. 141-148.

[2] A. Lindgren, Oluv Schelen, (2004) "Probabilistic Routing in Intermittently Connected Networks," Springer LNCS, Vol 3126. pp 239-254.

[3] A. Spyropoulos et al., (2005) "Spray and Wait: An Efficient Routing Scheme For Intermittently Connected Mobile Networks," ACM SIGCOMM Wksp. Delay Tolerant Networking (WDTN05).

[4] Alan Demers, Dan Greene, Carl Hauser, Wes Irish, John Larson, Scott Shenker, Howard. Sturgis, Dan Swinehart, and Doug Terry. (1987) "Epidemic algorithms for replicated database maintenance." In Proceedings of the 6th ACM Symposium on Principles of Distributed Computing, pp 1-12.

[5] Amin Vahdat and David Becker. (2000) "Epidemic Routing for partially connected ad hoc networks", Technical report CS- 200006, Duke University. http://issg.cs.duke.edu/epidemic/

[6] Anders Lindgren, Kaustubh S. Phanse, (2006) "Evaluation of Queuing Policies and Forwarding Strategies for Routing in Intermittently Connected Networks" in proceedings of IEEE COMSWARE '06, pp 1-10.

[7] Aruna Balasubramanian, Brian Neil Levine and Arun Venkataramani, (2007) "DTN Routing as a Resource Allocation Problem", ACM SIGCOMM, Computer Communication Review, Vol. 37, No.4.

[8] Bundle Protocol specification , (2007) IETF, RFC 5050.

[9] Evan P.C. Jones, Paul A.S.Ward, (2007) “ Practical Routing in Delay Tolerant Networks” , IEEE Transactions on Mobile Computing, Vol. 6, No. 8,pp. 943-959.

[10] J. Burgess, B. Gallagher, D.Jensen and B.N. Levine, (2007) "MaxProp: Routing for vehicleBased Disruption-Tolerant Networks", in Proceedings of IEEE Infocom, pp 1-11.

[11] Kevin Fall, (2007) “A delay-Tolerant Network Architecture for challenged Internet “, IETF, RFC 4838.

[12] Keranen, A. (2008) “Opportunistic Network Environment Simulator," Special Assignment report, Helsinki University of Technology, Department of Communication and Networking.

[13] Sushant Jain, Kevin Fall, Rabin Patra, (2004) "Routing in Delay Tolerant Network," in proceedings of ACM- SIGCOMM '04.

[14] TKK/COMNET. Project page of the ONE simulator. 2008 http://www/netlab.ttk.fi/tutkimus/dtn/theone,

[15] Zhensheng Zhang, San Diego Research Center , (2006) "Routing In Intermittently Connected Mobile AdHoc Networks And Delay Tolerant Networks: Overview and Challenges," IEEE Communications Surveys Tutorials, Vol. 8, No. 1. pp 24-37.

[16] A. Pentland, R.Fletcher and A.Hasson, (2004) "Daknet: Rethinking connectivity in developing nations," IEEE Computer,Vol. 37,No.1 pp.78-83.

[17] P. Basu and T.Little, (2002) "Networked parking spaces: architecture and applications," in IEEE vehicular Technology conference (VTC). 
International Journal of Wireless \& Mobile Networks (IJWMN) Vol. 3, No. 5, October 2011

[18] Amir Krifa, Chadi Barakat, Thrasyvoulous Spyropolous, (2008) “ An Optimal Buffer Management Policies for Delay Tolerant Networks", in Proceedings of IEEE SECON 2008, pp. 260-268.

[19] Ram Ramanathan, Richard Hansen, Prithwish Basu, Regina Rosales-Hain, Rajesh Krishnan, (2007) "Prioritized Epidemic Routing for Opportunistic Networks,” MobiOpp '07.

[20] David Hay, Paolo Giaccone, (2009) "Optimal Routing and Scheduling for Deterministic Delay Tolerant Networks", in Proceedings of the Sixth international conference on Wireless OnDemand Network Systems and Services- 2009

[21] Thrasyvoulous Spyropolous, Cauligi S. Raghavendra, (2008) "Efficient Routing in Intermittently Connected Mobile Networks: the Multi-Copy Case,” in IEEE/ACM Trans. On Networking, Vol 16. No.1 pp. 63-76.

[22] E.M.Daly, M.Haahr, (2010) "The Challenges of Disconnected Delay-Tolerant MANETs", Elsevier Ad Hoc Networks Journal, vol. 8, no. 2, pp. 241-250.

[23] Amir Krifa, Chadi Barakat, Thrasyvoulous Spyropolous, (2008) “An Optimal Joint Scheduling and Drop Policy for Delay Tolerant Networks”, IEEE WoWMoM -08, pp. 1-6.

[24] "Delay tolerant networking research group." [Online]. Available: http://www.dtnrg.org

[25] S. Farrell, V. Cahill, D. Geraghty, I. Humpreys and P. McDonald, (2006) " When TCP Breaks: Delay and Disruption- Tolerant Networking”, IEEE Internet Computing, Vol.10, No.4, pp 7278 .

[26] G.Fathima, Dr. R.S.D Wahidabanu, (2010). "Comparison of DTN routing protocols and their Buffer utilization using ONE simulator," in Proceedings of International Conference on Wireless Networks- WorldComp'10, Vol.1 pp 252-256.

\section{Authors}

G. Fathima obtained B.E degree in Computer Science \& Engineering from Madurai Kamaraj University, Madurai in 1994 and M.E in Computer Science from R.E.C., Trichirapalli in 2003. She joined as Lecturer in Adhiyamaan

College of Engineering, Hosur in 1997. Currently working as Asst.Professor in the Department of Information Technology. Her research interest includes analysis of routing protocols of Wireless and Mobile Ad hoc Networks. She is the life member of ISTE and IE.

Dr. R.S.D.Wahida Banu obtained B.E. degree in 1981 and her M.E. degree in Jan ' 85 from GCT, Coimbatore, Madras University. She got the Ph.D. degree in 1998 from Anna University, Chennai. She is an Engineering educator. To her credit, she has 28 years of Teaching experience which includes 16 years of rich research experience. She is the member of ISOC, IAENG, VDAT and life member of ISTE, IE, CSI and SSI. She is currently working as Professor and Head of Department of Electronics and Communication Engineering, Government College of Engineering, Salem.
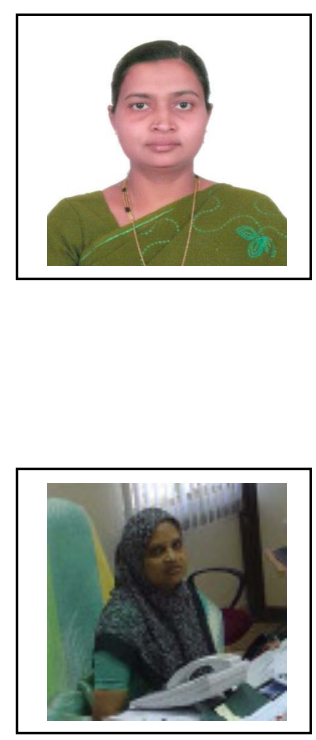